\title{
In Situ Analytical Electron Microscopy and Cryogenic Electron Microscopy for Characterizing Nanoscale Materials in Electrochemical Process
}

\author{
Minghao Zhang ${ }^{1}$, Xuefeng Wang ${ }^{1}$, and Ying Shirley Meng ${ }^{1 *}$ \\ 1. Department of NanoEngineering, University of California San Diego, La Jolla, USA \\ * Corresponding author: shmeng@ucsd.edu
}

Lithium-ion batteries (LIBs) commercially dominate portable energy storage and have been extended to hybrid/electric vehicles by utilizing electrode materials with enhanced energy density. However, the energy density and cycling life of LIBs must extend beyond the current reach of commercial electrodes to meet the performance requirements for transportation applications [1]. Carbon-based anodes, serving as the main negative electrodes in LIBs, have an intrinsic capacity limitation due to the intercalation mechanism [2]. Alternative anodes are being explored, including nanosized metal sulfides and oxides which were found to possess better cycling stability and larger reversible capacities [3]. Recently, researchers proposed to use Li metal as the anode to achieve even higher energy density [4]. However, the dendritic growth of Li metal during cycling will result in low coulombic efficiency as well as safety issue which is detrimental for practical applications.

Further improvements and developments of electrode materials rely on a fundamental understanding of their electrochemical cycling mechanisms. On the one hand, real-time (in situ) studies with high spatial resolution are required for characterizing microstructural evolution and specific electrochemical reactions upon cycling. In situ TEM coupled with electron energy loss spectroscopy (EELS) has thus been considered as one of the most powerful techniques for monitoring electrochemical processes in electrode materials [5]. EELS spectra interpretation is critical for chemical information analysis including oxidation state, charge transfer, bonding environment, etc. On the other hand, metal anode and solid electrolyte interphase (SEI) are chemically reactive and sensitive to electron-beam irradiation. TEM studies of batteries in their native environment can be problematic. Cryogenic electron microscopy (cryo-EM) can potentially be a powerful technique to image beam sensitive battery electrode materials [6].

In this work, the lithiation mechanism of nanosized metal sulfides and oxides is explored using in situ TEM. Multi-steps lithiation mechanism was revealed with discovery of a fascinating, spontaneously formed, self-assembling nanocomposite framework. This framework provides adequate room to accommodate the volume expansion during lithiation while ensuring enhanced contact among the active materials. Probing these dynamic evolution processes is not only necessary for fundamental understanding of electrochemical mechanisms, but also beneficial for designing novel materials in batteries with improved performance. EELS was conducted at different lithiation steps to investigate chemical changes during lithiation. EELS spectra simulation based on a variety of ab initio methods is introduced for chemical signature interpretation (see Figure 1).

This work will also cover recent advances on cryo-EM development for metal dendritic growth and SEI. The detailed structure of electrochemically deposited $\mathrm{Li}$ and the SEI composition at the nanoscale were revealed with minimized beam damage during imaging. The results show that the nucleation-dominated electrochemically deposited $\mathrm{Li}$ is amorphous, while some SEI is crystalline. Our findings not only illustrate the capabilities of cryogenic microscopy for beam (thermal)-sensitive materials but also yield 
crucial structural information on the Li metal deposition (see Figure 2).

References:

[1] M. Armand and J.-M. Tarascon, Nature 451 (2008), 652-657.

[2] P. Poizot, S. Laruelle, S. Grugeon, L. Dupont and J. Tarascon, Nature 407 (2000), 496-499.

[3] B. Kang and G. Ceder, Nature 458 (2009), 190-193.

[4] X.-B. Cheng, R. Zhang, C.-Z. Zhao, F. Wei, and J.-G. Zhang, Advanced Science 3 (3) (2016), 1500213.

[5] D. Qian, C. Ma, K. L. More, Y. S. Meng and M. Chi, NPG Asia Mater. 7 (2015), e193.

[6] X. Wang, Y. Li and Y. S. Meng, Joule 2 (2018) 1-10.

[7] We are grateful for financial support from the Assistant Secretary for Energy Efficiency and Renewable Energy, Office of Vehicle Technologies of the U.S. Department of Energy under the Battery500 Consortium. This research was also sponsored by the Materials Sciences and Engineering Division of the U.S. Department of Energy (DOE), Office of Basic Energy Sciences (BES), and was performed at the Center for Nanophase Materials Sciences (CNMS) at Oak Ridge National Laboratory (ORNL), which is a DOE Office of Science User Facility.
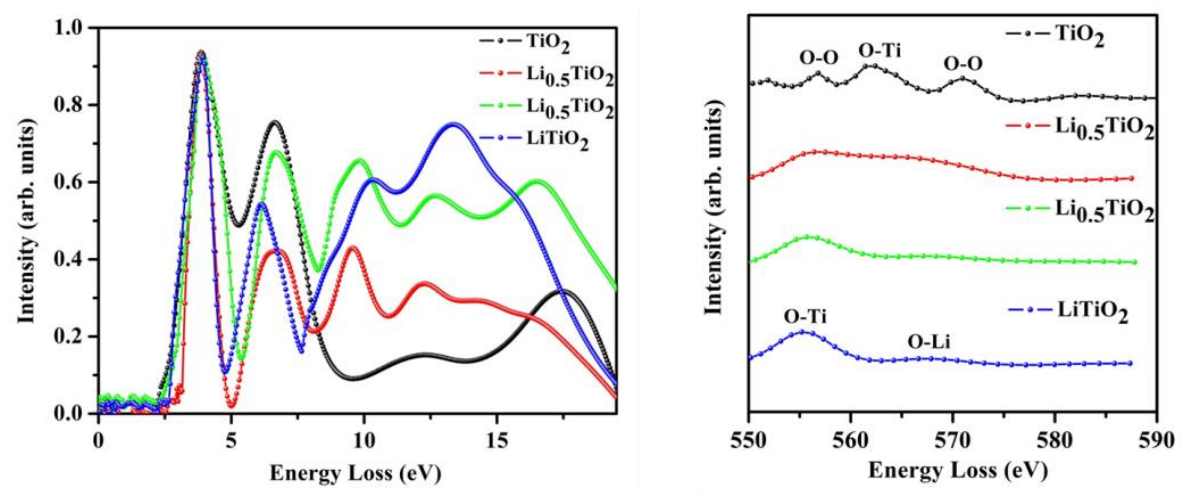

Figure 1. Oxygen K-edge ELNES simulation using WIEN2k package (left) and EXELFS simulation using FEFF9 package (right) for lithiation process of titanium oxides.
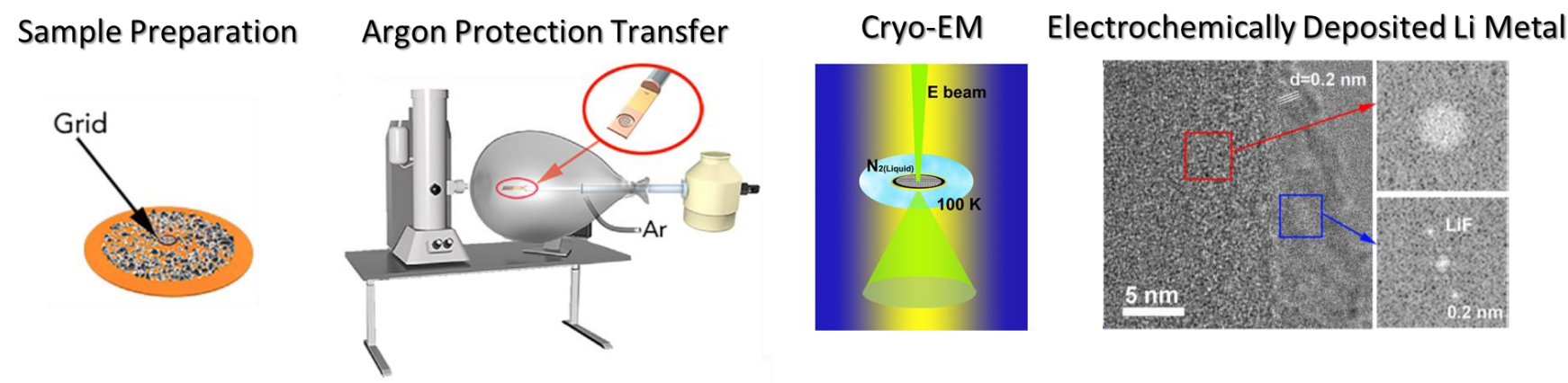

Figure 2. Schematic showing the process of cryo-EM characterization for electrochemically deposited Li metal. 